\title{
Current, new, and emerging therapies for managing hyperglycaemia in type 2 diabetes
}

The last decade has witnessed the introduction of several new drug classes and drug formulations that effectively reduce hyperglycaemia in type 2 diabetes. Expansion of drug choices is set against a background of increasing awareness of the progressive nature of type 2 diabetes. The next decade will see emergence of new therapies with novel modes of action further broadening the palate of agents available for clinical use. This expansion will enable tailoring of therapy in diabetes based on individual response to a particular drug, as well as any specific side-effects arising from treatment.

\section{CURRENT THERAPIES}

Consensus guidelines have now incorporated metformin as the first choice oral agent, along with appropriate dietetic and lifestyle advice, in the early management of hyperglycaemia in type 2 diabetes. ${ }^{1}$ It has also a role in preventing people with impaired glucose tolerance (IGT) progressing to type 2 diabetes. ${ }^{2}$ Metformin acts by reducing gluconeogenesis (elevated in type 2 diabetes) and improving insulin sensitivity. This is the only biguanide in current practice. Patients can experience significant gastrointestinal side effects which may be ameliorated by switching from immediaterelease preparations to newer delayedrelease formulations.

Recent NICE guidance promotes the use of sulphonylureas as second-line therapy. ${ }^{3}$ These agents act by directly stimulating insulin secretion by beta cells in a nonglucose dependent manner and can cause, in some individuals, weight gain and hypoglycaemia.

The place of thiazolidindiones - the 'TZDs' or 'glitazones' - has come into question following the publication of a metaanalysis showing an excess of myocardial infarctions in patients treated with rosiglitazone. ${ }^{4}$ As insulin sensitisers, whose action is mediated through peroxisome proliferator-activated receptor $\gamma$ (PPAR $\gamma$ ) activation, these drugs showed much promise in terms of durability of effect and, in those who respond well to the agent, useful reductions in $\mathrm{HbA1c}$. Pioglitazone has been shown to have beneficial effects on lipids and there appears to be established cardiovascular benefit, particularly in secondary cardiovascular prevention. ${ }^{5}$ Some concerns have been raised for the drug class about links to osteoporosis through a putative mechanism of 'uncoupling' of osteoblast and osteoclast action and this is subject to ongoing evaluation.

Insulin use in type 2 diabetes has increased considerably following publication of UKPDS. ${ }^{6}$ The introduction of analogue insulins, in either monophasic basal longacting formulations (insulin glargine, insulin detemir) or biphasic premixed preparations, have further facilitated uptake of insulin therapies. Analogue insulins, with their flatter kinetic profiles and reduced tendency to cause nocturnal hypoglycaemia, compare favourably with existing isophane insulins. This has made initiation and maintenance of insulin therapy in primary care more straightforward. However, considerable professional and patient education is still needed, as well as frequent blood glucose monitoring according to the frequency of insulin administration. In addition, some risk of hypoglycaemia remains and many patients with type 2 diabetes gain significant weight with insulin treatment with or without improved glycaemia.

\section{NEW THERAPIES}

The discovery of the 'incretin effect' has revealed a pathway in glucose homeostatis that can be targeted by new glucoselowering agents. This effect is seen when comparing insulin responses to intravenous and oral glucose loading in experimental situations, with a significantly greater insulin response being observed following the oral glucose. In those with normal physiology, ingestion of food results in the release of two incretin hormones, glucagon-like peptide-1 (GLP-1) from the $L$ cells lining the ileum, and glucose-dependent insulotropic polypeptide (GIP) from the $\mathrm{K}$ cells in the jejunum. In combination with neural signalling, the presence of incretin hormone results in pancreatic beta cell-receptor activation and insulin release. Importantly this process is glucose dependent as GLP-1 is not secreted in any significant quantity unless substrate is present in the bowel. GLP-1 is rapidly inactivated (1-2 minutes) by dipeptidyl peptidase-4 (DPP-4).

In people with type 2 diabetes GLP-1 secretion is markedly diminished, but GLP-1 receptor sensitivity preserved. ${ }^{7}$ By contrast, beta cell responsiveness to GIP is lost. Investigators have looked at methods of replacing GLP-1 or enhancing its action through inhibition of DPP-4. As a peptide, GLP-1 is destroyed in the stomach and cannot be given orally. Analogues, that are DPP-4 resistant, have been synthesised from reptilian peptide found in the saliva of the Gila monster (Heloderma suspectum) and share over $50 \%$ homology with native GLP-1.

The first incretin mimetic to have entered mainstream clinical use is exenatide. This twice-daily subcutaneous injection causes glucose-dependent insulin secretion, appetite suppression, delayed gastric emptying, and weight loss. ${ }^{8}$ As a result of its glucose-dependent action hypoglycaemia is uncommon. In early animal experiments increase in beta cell mass was reported. Exenatide has been available in the US for over 3 years and in the UK for the last 12 months. The main side effect is transient nausea in just under half of those treated, with approximately $3 \%$ discontinuation rate in the licensing trials. There have been 30 cases reported of pancreatitis worldwide but this is widely regarded as a background rate for such a population of patients. A oncedaily human GLP-1 analogue, liraglutide, will 
be commercially available in the near future. Liraglutide is albumin bound, exhibits $97 \%$ homology to human GLP-1, is DPP-4 resistant and has a half-life of 13 hours. ${ }^{9}$ In addition, a once-weekly long-acting release injectable preparation will shortly be added to the therapeutic range of incretin agents with fortnightly and monthly versions thereafter. ${ }^{10}$ Initial trials of an inhaled GLP-1 were reported at the recent American Diabetes Association Annual Scientific meeting.

Two drugs that inhibit the action of DPP-4 have been licensed in the UK and recently introduced into clinical practice. ${ }^{11}$ Sitagliptin is a once daily oral agent while vildagliptin is twice daily. Vildagliptin is also currently available in a fixed-dose formulation with metformin. Rises in liver function tests were seen with higher doses used in vildagliptin licensing trials and 3-monthly testing for the first year is recommended. These are generally slightly weaker glucose lowering agents than GLP-1, weight neutral, well tolerated and have the distinct advantage of being orally administered. Several other drugs in this class are coming to market. Long-term safety data for DPP-4 inhibitors is not yet available.

The impact of these newer agents on long-term cardiovascular risk remains uncertain. Recent very large studies (ADVANCE ${ }^{12}$ and ACCORD $^{13}$ ) using currently available therapies looked at the impact of intensification of blood glucose lowering on cardiovascular events, that is targets of $\leq 7 \%$. Neither trial showed statistically significant reductions in major cardiovascular events but both did show microvascular benefit. If trials were lengthened considerably, for example 10 years or more, early trends showing cardiovascular gain might become statistically meaningful. However, ACCORD was stopped because of an excess of deaths (predominantly due to ischaemic heart disease) in the intensively-treated group targeting $\mathrm{HbA} 1 \mathrm{c}<6 \%$. Subjects in ACCORD had longer duration of diabetes than ADVANCE, more cardiovascular complications and were treated with a more rapid glucose-lowering protocol (over a 3month period). They were on more oral and insulin therapies and had more hypoglycaemia. While the commonly used targets of $\mathrm{HbA} 1 \mathrm{c} 7 \%$ and $6.5 \%$ remain desirable in the management of type 2 diabetes the case, perhaps, aiming for $6 \%$ or lower requires some consideration while the issues raised by ACCORD are explored further.

\section{EMERGING THERAPIES}

There are several new classes of drugs targeting different aspects of glucose control.

Selective peroxisome proliferatoractivated receptor modulators (SPPARMs), similar in concept to selective oestrogen receptor modulators (SERMs), using adjuvant co-factors and producing less side effects than thiazolidinediones, are in a reasonably advanced stage of development. They will have an impact on glucose regulation and adipocyte function.

Sodium glucose co-transporter type 2 (SGLT2) inhibition is seen as a useful mechanism for improving hyperglycaemia. SGLT2 promotes $90 \%$ glucose reabsorption (along with sodium reabsorption) from the $\mathrm{S} 1$ section of the proximal renal tubule. Sodium glucose cotransporter Type 1 acts on the distal twothirds of the proximal tubule and re-absorbs $10 \%$ glucose. Inhibition of SGLT2 results in glycosuria and normalisation of blood glucose. Potential benefits include improved glucose control independent of insulin action, low risk of hypoglycaemia, use in both type 1 and type 2 diabetes, and improvements in blood pressure control consequent upon enhanced sodium excretion. Potential challenges with this drug class include polyuria, electrolyte imbalance, urinary tract infections, and dehydration. Dapagliflozin and remogliflozin are SGLT2 inhibitors in early human trials.

Glucokinase has been investigated as a potential therapeutic target. Through the dual action of glucose-stimulated insulin secretion and increased hepatic glucose uptake and utilisation, glucokinase activators improve glucose control. Potential problems include hypoglycaemia, weight gain, and fatty liver (steatosis).

In addition, it is recognised that people with type 2 diabetes have increased glucose production due to gluconeogenesis. Glucagon-receptor antagonists block the action of glucagon as well as increasing insulin and GLP-1 production. This action significantly attenuates hyperglycaemia in early human studies with reduction of
$\mathrm{HbA1c}$ and weight loss without hypoglycaemia. Potential problems include delayed recovery from hypoglycaemia, rebound hyperglucagonaemia, as well as direct liver and pancreatic effects.

Researchers looking for agents that delay ageing have identified that calorie-restricted rats live up to $25 \%$ longer than non-starved controls. Enhanced activity of enzymes belong to the sirtuin family has been shown to contribute to this longevity. Humans possess seven sirtuin deacetylases. Silent mating type information regulation 2 homolog Saccharomyces cerevisiae (SIRT1) simulates calorie restriction lowering blood glucose, improving insulin sensitivity and reducing insulin secretion. Resveratrol (SRT 501) (3,5,4'-trihydroxystilbene) is a polyphenolic phytoalexin and a SIRT1 activator. ${ }^{14}$ It is a natural product found in skin of red grapes and has been shown to reduce both glucose and insulin levels, decrease endothelial inflammation in the aorta, delay the onset of osteoporosis, and reverse the effects of a high calorie diet. SIRT 1 activating compounds (STACS) are in development and are a thousandfold more potent than resveratrol. ${ }^{15}$

\section{CONCLUSION}

Glucose lowering in type 2 diabetes continues to show clear benefits in terms of reduced microvascular risk. However with the established drug classes, such as sulphonylureas and insulin, glucose lowering is often at a therapeutic cost in the form of weight gain and hypoglycaemia. There are some uncertainties about the possible adverse effects of glitazones. GLP-1 analogues and DPP-4 inhibitors are glucosedependent agents that are either weight losing or weight neutral as well as far less likely to cause hypoglycaemia. ${ }^{16}$ However, longer-term data on durability and safety are awaited. Emerging therapeutic approaches to diabetes care appear to offer new opportunities for our patients to have individually-tailored therapy, achieve maximal glucose lowering, and avoid the important treatment side effects of hypoglycaemia and weight gain.

\section{Neil Munro,}

GP, Claygate, Surrey and Associate Specialist in Diabetes, Beta Cell Diabetes Centre, Chelsea and Westminster Hospital, London. 


\section{Michael D Feher,}

Consultant in Diabetes and Clinical Pharmacology, Clinical Lead for Diabetes, Beta Cell Diabetes Centre, Chelsea and Westminster Hospital, London.

\section{REFERENCES}

1. UK Prospective Diabetes Study (UKPDS) Group. Effect of intensive blood-glucose control with metformin on complications in overweight patients with type 2 diabetes (UKPDS 34). Lancet 1998; 352(9131): 854-865.

2. Knowler WC, Barrett-Connor E, Fowler SE, et al. Reduction in the incidence of type 2 diabetes with lifestyle intervention or metformin. N Engl J Med 2002; 346(6): 393-403.

3. National Collaborating Centre for Chronic Conditions. Type 2 diabetes. National clinical guidelines for managmen in primary and secondary care (update). http://www. nice.org.uk/nicemedia/pdf/CG66diabetesfullguideline.pdf (accessed 14 Jul 2008).

4. Nissen SE, Wolski K. Effect of rosiglitazone on the risk of myocardial infarction and death from cardiovascula causes. N Engl J Med 2007; 356(24): 2457-2471.

5. Erdmann E, Dormandy JA, Charbonnel B, et al. The effect of pioglitazone on recurrent myocardial infarction in 2445 patients with type 2 diabetes and previous myocardial infarction: results from the PROactive (PROactive 05)
Study. J Am Coll Cardiol 2007; 49(17): 1772-1780.

6. UK Prospective Diabetes Study (UKPDS) Group. Intensive blood-glucose control with sulphonylureas or insulin compared with conventional treatment and risk of complications in patients with type 2 diabetes (UKPDS 33). Lancet 1998; 352(9131): 837-853.

7. Nauck M, Stöckmann F, Ebert R, Creutzfeldt W. Reduced incretin effect in type 2 (non-insulin-dependent) diabetes. Diabetologia 1986; 29(1): 46-52.

8. Levy JC. Therapeutic intervention in the GLP-1 pathway in type 2 diabetes. Diabet Med 2006; 23(Suppl 1): 14-19.

9. Vilsbøll T, Brock B, Perrild H, et al. Liraglutide, a once-daily human GLP-1 analogue, improves pancreatic B-cell function and arginine-stimulated insulin secretion during hyperglycaemia in patients with type 2 diabetes mellitus. Diabet Med 2008; 25(2): 152-156.

10. Kim D, MacConell L, Zhuang D, et al. Effects of onceweekly dosing of a long-acting release formulation of exenatide on glucose control and body weight in subjects with type 2 diabetes. Diabetes Care 2007; 30(6): 1487-1493.

11. Richter B, Bandeira-Echtler E, Bergerhoff K, Lerch CL. Dipeptidyl peptidase-4 (DPP-4) inhibitors for type 2 diabetes mellitus. Cochrane Database Syst Rev 2008; (2): CD006739.

12. ADVANCE Collaborative Group, Patel A, MacMahon S, Chalmers J, et al. Intensive blood glucose control and vascular outcomes in patients with type 2 diabetes. $N$ Engl J Med 2008; 358(24): 2560-2572.
13. Action to Control Cardiovascular Risk in Diabetes Study Group, Gerstein HC, Miller ME, Byington RP, et al. Effects of intensive glucose lowering in type 2 diabetes. $N$ Engl J Med 2008; 358(24): 2545-2559.

14. Jiang W J. Sirtuins: novel targets for metabolic disease in drug development. Biochem Biophys Res Commun 2008: doi:10.1016/j.bbrc.2008.06.048.

15. Nayagam VM, Wang X, Tan YC, et al. SIRT1 modulating compounds from high-throughput screening as antiinflammatory and insulin-sensitizing agents. J Biomo Screen 2006; 11(8): 959-967.

16. Feher M, Cox A, Munro N. Management of hyperglycaemia in type 2 diabetes: a clinician's algorithm. Br J Diab Vas Dis 2008; 8: 3-4.

DOI: 10.3399/bjgp08X319684

ADDRESS FOR CORRESPONDENCE

\section{Neil Munro}

Beta Cell Diabetes Centre, 2nd Floor, Chelsea \& Westminster Hospital, 396 Fulham Road, SW10 9NH

E-mail: neil.m.munro@btinternet.com

\section{Reducing the rise in type 2 diabetes}

The York and Humber Public Health Observatory $^{1}$ reports that $4.7 \%$ of the population now have diabetes, of which $92 \%$ (4.3\% of the population) have type 2 diabetes. The diagnosed prevalence has doubled between 1994 and 2003. The increasing number of people with type 2 diabetes reflects several factors: an ageing population; a true increase in prevalence, related to increased prevalence of overweight and obesity; decreasing physical activity; better survival of those with diabetes due to improved control of blood glucose, blood pressure, and lipids; and earlier diagnosis, partly from informal screening. (A recent study using British general practice data found that one-third of people aged over 40 years [excluding those known to have diabetes] underwent blood glucose measurement in the previous 2 years. ${ }^{2}$ )

The debate on whether there should be organised screening has shifted towards a broad-based approach of cardiovascular risk reduction. This is logical, as the main risk for those with undiagnosed type 2 diabetes is vascular disease. The arguments for screening are that:

- Many people with type 2 diabetes, perhaps $20 \%$ although some estimates are higher, are undiagnosed.

- People can develop complications such as eye disease, before they develop symptoms and have their diabetes diagnosed.

- The risk of heart disease is increased, and the first symptom of heart disease may be a heart attack which is often fatal.

- People with impaired glucose tolerance (IGT) are also at higher risk of heart disease than those with normal glucose levels. ${ }^{3}$

- Progression to diabetes can be reduced or delayed by lifestyle measures (and by drug therapy, such as metformin).

So the aim of screening would be to treat hyperglycaemia and the vascular risk at an early stage, and to prevent complications. ${ }^{4}$

There is then the additional debate about whom to screen. Consensus exists on the need for selective screening, by age and risk factors. For practical purposes (given that screening would be done in primary care), the other factors would be those which are: firstly, recorded in GP records, and secondly, are strongly predictive of diabetes or 'prediabetes'. So screening might be based on: age over 40 years; body mass index (BMI) over $26 \mathrm{~kg} / \mathrm{m}^{2}$; presence of other markers of metabolic disease such as hypertension, high cholesterol or triglycerides, ischaemic heart disease, or peripheral arterial disease; and, if available, family history of diabetes or other metabolic disease. In reality, age and BMI provide a good basis for selection. ${ }^{5}$

If we did have organised screening, we would, depending on the test used and the cut-offs chosen, detect more people with lesser degrees of glucose impairment, such as IGT, than with undiagnosed diabetes. Macrae and colleagues ${ }^{6}$ screened high-risk patients and found $8.5 \%$ to have type 2 diabetes, and $9.6 \%$ to have IGT or impaired fasting glycaemia. In the wider population, there are probably about three times as many people with IGT as with type 2 diabetes. Non-pharmacological interventions with diet and physical activity have been shown to be effective in large trials in Finland, the US, India, and China. But these trials involved volunteers in whom compliance is likely to be much better than average, and even in them compliance waned over time, despite continued intervention. In other studies, once the intervention stopped, the effect was soon lost.

The duration of many trials is far too short 